\title{
Colliding Stellar Winds in Close Binary Systems
}

\author{
STEVEN N. SHORE \\ GHRS Science Team/CSC, GSFC, Code 681, Greenbelt MD 20771 USA \\ and \\ MICHAEL J. CORCORAN \\ Laboratory for High Energy Astronomy, GSFC, Code 660, Greenbelt MD 20771 USA
}

\begin{abstract}
.
We describe some of the observational phenomena associated with colliding stellar winds, focussing especially on close WR+O binaries. In particular, we report some new ultraviolet observations of CQ Cep and V444 Cyg. We also discuss some of the general theory of such wind collisions.
\end{abstract}

Key words: Stellar Winds - Hydrodynamics - Ultraviolet Spectroscopy

\section{Introduction}

In his thesis, Cherepashchuk (1967) presented the first statement of the possible existence of stellar wind collisions in close binary systems containing Wolf-Rayet components (see also Cherepashchuk 1990). We now see such interactions as one of a broad range of phenomena associated with binary star evolution. Heap (1981) proposed the application of the colliding wind model to the close (non-WR) binary HD 47129. Although not strictly a wind-wind interaction, Chapman (1981) argued for accretion wakes being present in $\zeta$ Aur systems. Girard and Willson (1987) proposed that symbiotic winds are partially structured by the interaction between the degenerate and the evolved companion. Koenigsberger and Auer (1985) and Shore and Brown (1988) have discussed the evidence for such interactions in WR+O systems. Gies et al. (1991) have suggested that AO Cas and other close O star binaries also show evidence for wind collisions. Most recently, Sahade and Brandi (1991) have re-discussed both AO Cas and HD 47129 in light new ultraviolet observations. In short, the phenomenon of dynamical interactions between mass losing stars in close binary systems cuts across a broad range of phenomenological classes.

\section{General Theoretical Picture}

The simplest picture of wind collisions can be obtained from the calculation of the shape of the contact discontinuity between the two winds. The collision region is marked by a stand-off shock on either side, one concave toward the $\mathrm{O}$ star and the other convex toward the WR star, whose wind is assumed to be the stronger. The interaction of two winds moving at terminal speed produces an equilibrium at a point $\Delta r=a\left(1+\lambda^{1 / 2}\right)^{-1}$, where $\lambda \equiv \dot{M}_{2} v_{\infty, 2} / \dot{M}_{1} v_{\infty, 1}, \dot{M}$ is the mass loss rate, $v_{\infty}$ is the terminal velocity for each of the stars, and $a$ is the separation of the centers. Along the contact surface, there will be an active acceleration of material away from the line of centers. This flow accelerates through the sonic point, forming a Mach collar around the central collision zone at which the temperatures peak. The expansion manifests itself by a high velocity tail in the resonance line profiles when the system is viewed down the axis of the cylindrically symmetric shock. The 
analytic solution for the region in the $(\xi, \eta)$ coordinate system, with $\xi$ along the line ul centers, is:

$$
\frac{d \eta}{d \xi}=\frac{1}{2}\left[A_{1} \sin 2 \alpha_{1}+A_{2} \sin 2 \alpha_{2}\right] /\left[A_{1} \sin ^{2} \alpha_{1}-A_{2} \sin ^{2} \alpha_{2}\right]
$$

where $A_{1,2}=\dot{M}_{1,2} v_{1,2} / 4 \pi r_{1,2}^{2}, \sin \alpha_{1}=\xi / r_{1}$, and $\sin \alpha_{2}=(a-\xi) / r_{2}$ (Shore and Brown 1988, Luo et al. 1990, Stevens et al. 1991). Using typical wind laws (either terminal winds where $v_{1,2}=v_{\infty, 1,2}$ or schematic radiative winds where $v_{1,2}=$ $v_{\infty, 1,2}\left(1-R_{\star, 1,2} / r\right)^{\beta}$ with $\left.\beta \approx 1\right)$ give reasonable solutions for the shape of the contact surface for distances $r_{1}$ and $r_{2}$ from each of the stars.

The detailed dynamics of the post-shocked material along the shock front is more complicated to model. In part this problem results from the long relaxation time required for calculations in the presence of thermal and hydrodynamic instabilities. The contact surface is Kelvin-Helmholtz unstable and therefore likely degrades into turbulence. Post-shock temperatures in excess of $10^{7} \mathrm{~K}$ are expected from both dynamic (wind-wind) and bow (wind-blunt body) collisions. The $\mathrm{x}$-ray emission depends on the details of the escape of photons because the collision occurs deep within the WR wind. Absorption and scattering should considerably alter any $\mathrm{x}$-ray signature (Luo et al. 1990, Stevens et al. 1991) and produce phase modulation of the emission.

\section{WR+O Binaries: CQ Cephei and V444 Cygni}

Earlier UV and optical studies of the short period WR+O binaries CQ Cep and V444 Cyg have noted the possibilities of wind-wind interactions.

Stickland et al. (1984) have shown that the O star in CQ Cep (WN7+O, $\mathrm{P}=1 .^{d} 64$ ) must have a large radius and is likely not an active wind source. They chose to model the interaction in terms of a possible standing shock near the O star surface. The interaction is very similar to that first discussed by Prilutskii and Usov (1976). A bow shock at about $1.25 \mathrm{R}_{\star}$ heats the gas, producing $\mathrm{x}$-rays. Although Prilutskii and Usov did not specifically discuss CQ Cep, we argue here and in Shore and Corcoran (1991, in preparation) that the UV observations are more consistent with this interpretation, possibly associated with a frustrated wind from the $\mathrm{O}$ star, than with an active wind-wind collision.

V444 Cyg (WN5+O6-8III, $\mathrm{P}=4 .^{d} 42$ ) has been the object of many studies, both UV, optical, and near infrared. The photometric analysis by Eaton, Cherepashchuk, and Khalliullin (1985) determined that the atmosphere is extensive and that the $O$ star is relatively small compared with the separation of the stars. Koenigsberger and Auer (1985), in discussing the behavior of the UV spectrum, argued that the system might display some of the symptoms of wind collisions. Underhill and Fahey (1987) proposed an alternative explanation for the UV resonance line profile variations in terms of confined magnetic loops, a view that has not been well supported by subsequent studies but one that called attention to the non-spherical nature of the WR and O star winds. Shore and Brown (1988), in a high resolution IUE study, showed that the line profile variations fit well the expectations of a colliding wind model of the sort outlined in section 2. They showed that the $\mathrm{O}$ star wind sculpts 
out a cavity in the WR wind. This study showed that near secondary eclipse, when the O star occults the WR star, the disappearance of the P Cyg absorption on the N IV $\lambda 1718$ and He II $\lambda 1640$ lines and the appearance of a high velocity extension on the C IV $\lambda 1550$ profile are all consistent with a dynamical interaction of the two stellar winds.

It is possible that the WC6-7+O5 system HD $152270\left(P=8{ }^{d} 89\right)$ also displays the optical signatures of wind-wind collisions. Neutsch, Schmidt and Seggewiss (1981) have argued on the basis of the C. III $\lambda 5696$ that the O star blows out a portion of the WR wind. We have examined all available high dispersion IUE spectra for this star. Although the line profiles show phase-dependence, there is nothing analogous to the effect seen in V444 Cyg on any of the profiles. More likely the WR wind is ionized by and structured by the $\mathrm{O}$ star in a way more similar to CQ Cep, that is by non-dynamical interaction.

We have recently obtained IUE high resolution observations of CQ Cep and V444 Cyg with IUE. The spectra were taken within only a few orbits in order to minimize the secular variability that might be associated with instabilities of the intra-system flows. The C IV profiles for CQ Cep are shown in Fig. $1 a$ and those for V444 Cyg are shown in Fig. 1b. Notice that the CQ Cep profiles vary in shape in a manner that indicates that the terminal velocity of the WR wind is altered and that the amount of material along the line of sight has been decreased, but the high velocity tail evident on the C IV profiles for V444 Cyg are absent. The N IV and He II profiles show similar behavior. The radial velocities track the WR star in both systems. The previous conclusions of Shore and Brown (1988) for V444 Cyg are confirmed and extended by the new data set. The interaction is not symmetric about secondary minimum but trails the $\mathrm{O}$ star indicating that the wind cavity is aberrated by orbital motion. Clearly a full three dimensional treatment of the interaction is required to understand the details of the interaction in this system. X-ray variations have previously been reported on the basis of EINSTEIN observations by Moffatt et al. (1982). These observations are an important window into the interior of the shock region. We have also obtained simultaneous ROSAT observations during the 1991 IUE campaign on V444 Cyg, but these data have not yet been received.

\section{Acknowledgements}

SNS thanks the AAS for travel support to attend this symposium and NASA for continued support for IUE research. We thank Sally Heap, Tom Bolton, Albert Petschek, Larry Libersky, Doug Brown, and Nichol St. Louis for discussions.

\section{References}

Chapman, R. D. 1981, ApJ, 248, 1043.

Cherepashchuk, A. M. 1967, Variable Stars, 16, 226;

Cherepashchuk, A. M. 1990, Astro. Zh., 67, 955.

Eaton, J., Cherepatshchuk, A., and Khalliullin, Kh. 1985, ApJ, 281, 774.

Gies, D. and Wiggs, S. 1991, ApJ, in press.

Girard, T. and Willson, L. A. 1987, $A \& A, 183,247$. 

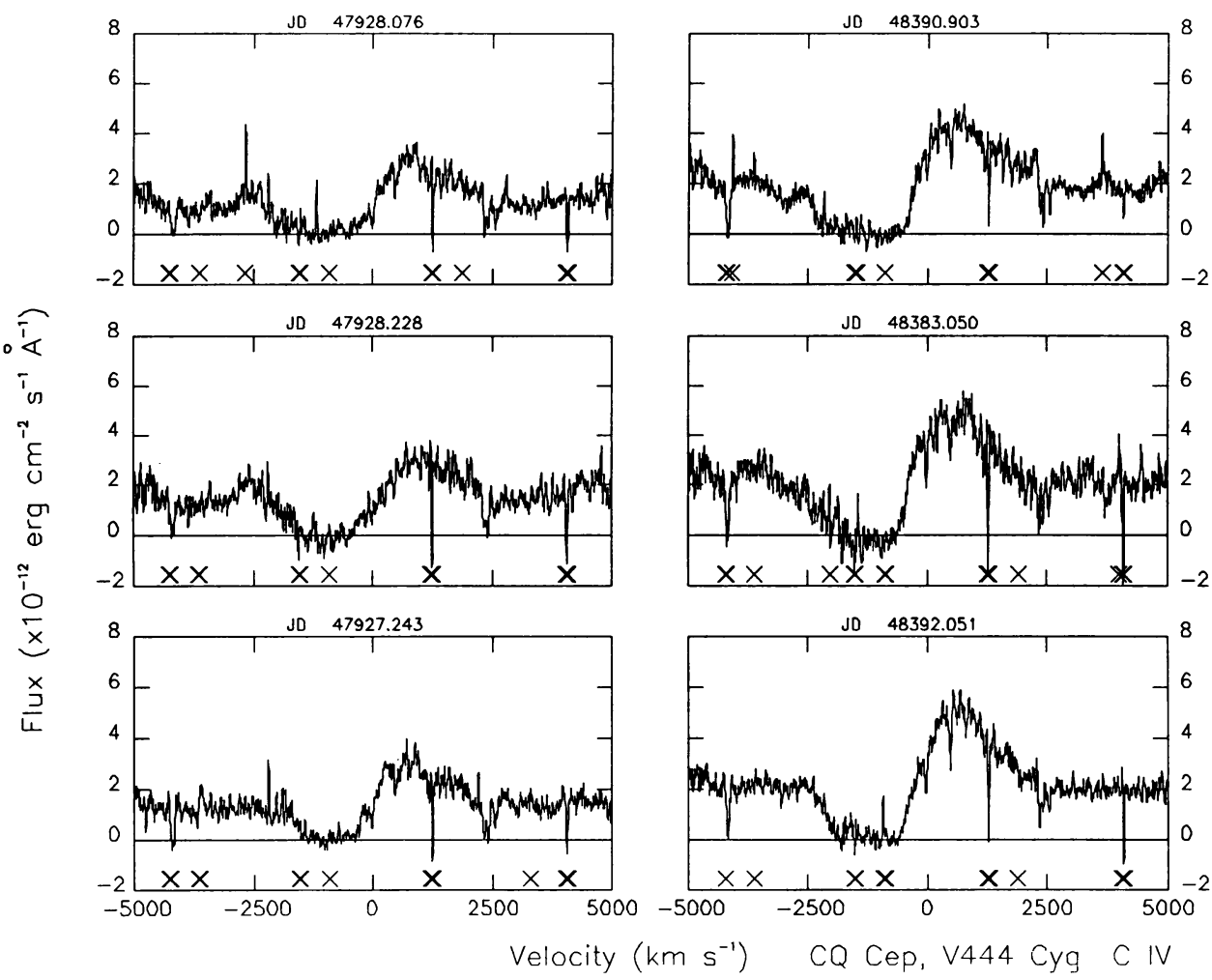

Fig. 1. a. CQ Cep, b. V444 Cyg. Examples of C IV line profiles at comparable phases (Shore and Corcoran 1991, in preparation). Top to bottom, phases $0.5,0.7$, and approx. 1.0 .

Heap, S. R. 1981, in The Universe at Ultraviolet Wavelengths: The First Two Years of the International Ultraviolet Explorer (ed. Chapman, R. D.) (NASA CP-217), p. 485.

Koenigsberger, G. and Auer, L. 1985, ApJ, 297, 255.

Luo, D., McCray, R. , and Mac Low, M. 1990, ApJ, 362, 267.

Moffatt, A., Firmani, F., McLean, I. S., and Segewiss, W. 1982, in IAU Symp. 99: Wolf-Rayet Stars: Observations, Physics, and Evolution (eds. de Loore, C. and Willis, A. J.) (Dordrecht: Reidel) p. 577.

Neutsch, W., Schmidt, H., and Seggewiss, W. 1981, Acta. Astr., 31, 197.

Prilutskii, O. and Usov, V. 1976, Sov. Astr. - AJ, 20, 2.

Sahade, J. and Brandi, E. 1991, ApJ, 379, 706.

Shore, S. N. and Brown, D. N. 1988, ApJ, 334, 1021.

Stevens, I. R., Blondin, J. M., and Pollack, A. M. T. 1991, ApJ, in press.

Strickland, D. J., Bromage, G. E., Budding, E., Burton, W. M., Howarth, I. D., Jameson, R., Sherrington, M. R., and Willis, A. J. 1984, $A \& A, 134,45$.

Underhill, A. B. and Fahey, R. 1987, ApJ, 313, 358. 